\title{
REFLEXIONES SOBRE LA SOSTENIBILIDAD DE LOS SERVICIOS PÚBLICOS, UN NUEVO PRINCIPIO GENERAL EN GESTACIÓN
}

\author{
TOMÁs-RAMÓN FERNÁNDEZ \\ Catedrático de Derecho Administrativo \\ Universidad Complutense de Madrid
}

\begin{abstract}
RESUMEN
La idea de sostenibilidad está bien afirmada en el ordenamiento ambiental y urbanístico, pero la crisis económica de la que no acabamos de salir y los compromisos internos y externos que hemos asumido para hacerla frente obligan a pensar en la necesidad de extenderla al conjunto de los servicios y actividades de las Administraciones públicas.
\end{abstract}

Palabras clave: sostenibilidad; crisis económica; servicios públicos; actividad administrativa.

\begin{abstract}
The idea of sustainability is well established in the environmental and urban legal system, but the economic crisis that we are not able to overcome and internal and external commitments engaged to face it force us to think about the need to extend it to all services and activities of the Public Administrations.
\end{abstract}

Key words: sustainability; economic crisis; public services; administrative activity. 


\section{SUMARIO}

I. INTRODUCCIÓN.-III. LA SOSTENIBILIDAD AMBIENTAL COMO PRINCIPIO INFORMADOR DE LA ORDENACIÓN TERRITORIAL Y URBANÍSTICA.-III. LA EXIGENCIA ADICIONAL DE SOSTENIBILIDAD ECONÓMICA.-IV. LA NECESARIA EXTENSIÓN AL CONJUNTO DE LOS SERVICIOS Y ACTIVIDADES DE LAS ADMINISTRACIONES PÚBLICAS DEL PRINCIPIO GENERAL DE SOSTENIBILIDAD FINANCIERA.

\section{INTRODUCCIÓN}

Hace unos meses en una conferencia que tuve el honor de pronunciar en la Academia Matritense del Notariado comenzaba yo recordando que las modas juegan también en el derecho en general y, en particular, en el derecho público un papel nada desdeñable al poner en primer plano ideas y preocupaciones que responden, ciertamente, a necesidades reales que en un momento dado se presentan como perentorias y atraen por ello la atención de todos ${ }^{1}$.

En una sociedad tan vertiginosa y tan estridente como la actual las modas en este ámbito tan singular que es el nuestro tienen siempre algo de inquietantes porque cuando surgen uno no puede estar nunca seguro de si se trata de algo pasajero, como muchas veces sucede, o de si, por el contrario, debajo de la densa y llamativa capa de retórica que las envuelve, puede haber algo valioso, destinado a dejar una huella, mayor o menor, en el sistema y merecedor por ello de atención desde el primer momento, aunque con el paso del tiempo pueda perder su fulgor inicial.

La participación, que hizo furor en la década de los «setenta» del pasado siglo, ilustra muy bien lo que quiero decir. La moda pasó ya hace mucho tiempo de forma que hoy ya no es necesario, ni tan siquiera apropiado, poner el énfasis de entonces al pronunciar la palabra porque la idea que expresa se ha integrado perfectamente en el sistema como una pieza más del mismo. Nadie cree hoy, por supuesto, que la participación pueda producir efectos milagrosos de ningún tipo, pero nadie se atrevería tampoco a prescindir de ella en este momento. Comenzó siendo una moda, pero ha terminado siendo un principio general que la Administración debe respetar en su actuación.

En el escenario actual del derecho público dominan hoy dos palabras, sin las cuales no parece posible dar un solo paso: transparencia y sostenibilidad. A la primera de ellas, que se encaramó al título de varios

1 La conferencia fue pronunciada el 19 de noviembre de 2015 y versó sobre La transparencia en la contratación administrativa. La revista Notario, en su núm. 65 correspondiente a enero-febrero de 2016, publicó un amplio resumen de la misma. 
centenares de normas antes de dar vida a la aparatosa Ley 19/2013, de 9 de diciembre, de Transparencia, Acceso a la información pública y Buen gobierno, hube de referirme en la ocasión recordada al comienzo, aunque ciñéndome al ámbito concreto que mis anfitriones me habían propuesto, la contratación administrativa ${ }^{2}$. A la segunda, que tiene ya un arraigo indiscutible en el derecho ambiental y urbanístico, quiero dedicar ahora unas breves reflexiones, porque en estos últimos años y a consecuencia de la brutal crisis económica y financiera cuyas consecuencias seguimos padeciendo todavía aparece por doquier con razón o sin ella dando nombre a las Leyes más diversas desde la cuasiconstitucional Ley Orgánica de Estabilidad Presupuestaria y Sostenibilidad Financiera de 27 de abril de 2012 a la Ley 27/2013, de 27 de diciembre, de Racionalización y Sostenibilidad de la Administración Local, pasando por la inefable Ley de Economía Sostenible de 4 de marzo de 2011, en la que hay un poco de todo, por la Ley de 27 de diciembre de 2012, de Medidas fiscales para la sostenibilidad energética e, incluso, por la Ley $2 / 2013$, de 29 de mayo, de Protección y uso sostenible del litoral y de modificación de la Ley 22/1988, de 28 de julio, de Costas.

¿Es sólo una concesión retórica a algo que se considera políticamente correcto en tiempos de crisis? ¿Hay algo en común que pueda predicarse con fundamento en ámbitos tan heterogéneos? ¿Pasará la fiebre cuando la tempestad económica se calme o estamos asistiendo a la gestación de un nuevo principio general aplicable como tal a la actividad de las Administraciones públicas? A estas preguntas intentaré dar una respuesta a continuación, para lo cual comenzaré, como es lógico, por lo que constituye el núcleo originario en el que la palabra cobró vida.

\section{LA SOSTENIBILIDAD AMBIENTAL COMO PRINCIPIO INFORMADOR DE LA ORDE- NACIÓN TERRITORIAL Y URBANÍSTICA}

Hasta el momento de nuestro ingreso en las Comunidades Europeas, hace ya treinta años, que es ahora nuestra referencia temporal, el derecho español apenas se había asomado a la problemática ambiental, que, por lo demás, había comenzado a manifestarse apenas quince años antes, al comienzo de la década de los «setenta».

2 Para el proceso de afirmación del principio de transparencia, vid. T. R. Fernández (2002), «El acceso de los ciudadanos a la información pública. Del derecho individual de defensa al principio general de transparencia», en P. ABERASTURY y H. J. Blanke (coords.), Tendencias actuales del procedimiento administrativo en Latinoamérica y en Europa (págs. 156 y ss.), Buenos Aires: Eudeba, K. Adenauer Stiftung. 
Al producirse la integración tuvimos, pues, que incorporar el acervo comunitario y con él la técnica de la evaluación del impacto ambiental que regulaba entonces la Directiva 85/377/CEE, de 27 de junio, lo que se llevó a cabo por el Real Decreto legislativo 1302/1986, de 28 de junio, uno más de los muchos que hubieron de aprobarse en el primer semestre de 1986 al amparo de la amplia delegación otorgada con ese fin por la Ley de Bases de 27 de diciembre de 1985.

Apenas un año después apareció el célebre Informe Bruntland que acertó a acuñar la expresión «desarrollo sostenible» y a definir éste con una sencillez y una precisión insuperables como «aquel desarrollo que cubre las necesidades de las generaciones presentes sin comprometer la capacidad de las generaciones futuras para satisfacer las suyas».

Fue éste, sin duda, un hallazgo feliz porque por mucho que se haya criticado luego su supuesta ambigüedad es lo cierto que en aquel momento consiguió expresar con apenas una veintena de palabras un programa de acción en materia territorial y ambiental que ha calado profundamente en nuestro ordenamiento urbanístico, en el que tiene una amplia acogida tanto en la legislación estatal, esto es, en el vigente Texto Refundido de la Ley de Suelo y de Rehabilitación urbana de 30 de octubre de 2015 como en la legislación autonómica ${ }^{3}$.

No voy a intentar, ni mucho menos, exponer aquí el alcance del principio de desarrollo territorial y urbano sostenible que hoy consagra el artículo 3 del citado Texto Refundido, ni, por supuesto, las reglas concretas a las que ha dado vida, pero sí quiero resaltar unas cuantas ideas que puedan ayudarnos luego a comprender mejor lo que el concepto de sostenibilidad puede dar de sí desde una perspectiva más general, que es lo que en este momento me interesa especialmente.

La primera y fundamental concierne a la concienciación acerca de la imperiosa necesidad de hacer un uso racional y, por lo tanto, limitado de los recursos naturales y, en particular, de los no renovables, entre los cuales está, obviamente, el suelo, cuya ocupación ha de ser eficiente y deberá restringirse a lo «preciso para satisfacer las necesidades que lo justifiquen» (artículos 3.3 y 20.1).

Hay aquí una apuesta decidida a favor de un modelo de ciudad compacta, así como de la regeneración del tejido urbano existente, opciones ambas que pretenden reducir el consumo de suelo y los mayores costes

3 J. C. Allí Aranguren y Allí Turrillas (2008), Estudio sistemático del Texto Refundido de la Ley de Suelo, Madrid: Dykinson, Gobierno de Navarra, Instituto Navarro de Administración Pública, dedican el capítulo III de la obra a El desarrollo territorial y urbano sostenible, págs. 157 a 242, con un nivel de detalle casi monográfico. 
que suponen las urbanizaciones extensivas, tanto para la implantación inicial de los servicios, como para su mantenimiento ulterior.

La Exposición de Motivos del texto inicial de la Ley de Suelo de 28 de mayo de 2007, que el Texto Refundido de 20 de julio de 2008 conservó, contiene un texto muy expresivo en el que, tras afirmar que «el urbanismo español contemporáneo es una historia desarrollista, volcada sobre todo en la creación de nueva ciudad», añade que, si bien el crecimiento urbano sigue siendo necesario, hoy «el urbanismo debe responder a los requerimientos de un desarrollo sostenible, minimizando el impacto de aquel crecimiento y apostando por la regeneración de la ciudad existente. La Unión Europea - dice- insistió claramente en ello, por ejemplo en la Estrategia Territorial Europea o en la más reciente Comunicación de la Comisión sobre una Estrategia Temática para el Medio Ambiente Urbano, para lo que propone un modelo de ciudad compacta y advierte de los graves inconvenientes de la urbanización dispersa o desordenada: impacto ambiental, segregación social e ineficiencia económica por los elevados costes energéticos, de construcción y mantenimiento de infraestructuras y de prestación de servicios públicos. El suelo - concluye-, además de un recurso económico, es también un recurso natural, escaso y no renovable».

Es una lástima que el Texto Refundido vigente de 30 de octubre de 2015 haya prescindido de este pasaje que tan bien definía la nueva política urbanística y tan clara dejaba su raíz europea ${ }^{4}$, que ha prendido con fuerza en las Leyes autonómicas, todas las cuales sin excepción insisten en la idea del desarrollo sostenible y no conformes con eso establecen límites de sostenibilidad fijando, incluso, porcentajes de superficie edificable en función del tamaño de los municipios, como hace la novísima Ley gallega 2/2016, de 10 de febrero, siguiendo la senda de la anterior Ley 9/2002, de 30 de diciembre, o remitiendo a los planes generales su determinación, como la Ley de Cantabria de 25 de junio de 2001 o, en fin, consagran expresamente en unos u otros términos el modelo de ocupación de suelo basado en la idea de ciudad compacta. Así lo hacen,

\footnotetext{
${ }^{4}$ Las refundiciones suelen llevarse por delante las exposiciones de motivos de las Leyes que se refunden, lo cual deja huérfano de explicación el contenido del texto resultante. La pérdida es sensible en todo caso, pero hay ocasiones en que la desaparición del preámbulo de una Ley es especialmente lamentable por la extraordinaria calidad de éste. Estoy pensando concretamente en el preámbulo del texto inicial de la Ley de Aguas de 3 de agosto de 1985 cuyo primer párrafo era de una calidad tan absolutamente inusual que no me resisto a transcribirlo aquí, aunque no tenga nada que ver con el asunto que ahora nos ocupa: «El agua es un recurso natural escaso, indispensable para la vida y para el ejercicio de la inmensa mayoría de las actividades económicas; es irremplazable, no ampliable por la mera voluntad del hombre, irregular en su forma de presentarse en el tiempo y en el espacio, fácilmente vulnerable y susceptible de usos sucesivos».
} 
por ejemplo, la Ley de Urbanismo de Aragón de 17 de junio de 2009 en su artículo 39.a), la Ley Foral 5/2015, de 5 de marzo, de medidas para favorecer el urbanismo sostenible y la actividad urbanística en Navarra, artículo 51 bis, la Ley Balear 2/2014, de 25 de marzo, de Ordenación y uso del suelo, artículo 3.2, y la Ley 7/2014, de 12 de septiembre, de Medidas sobre Rehabilitación, Regeneración y Renovación Urbana y sobre Sostenibilidad, Coordinación y Simplificación en materia de urbanismo de Castilla y León, cuya Exposición de Motivos dice con acierto que «en pocos años el concepto de sostenibilidad se ha instalado en el centro del debate urbanístico y es hoy un elemento imprescindible en los procesos de planificación» y que el criterio del crecimiento compacto, que «es ya protagonista del debate y la práctica del urbanismo comprobadas sus ventajas sociales frente al desarrollo disperso, permite optimizar servicios e infraestructuras... y preservar la identidad cultural que se plasma en un modelo de crecimiento planificado y sostenible».

La idea de sostenibilidad ambiental está, por lo demás, bien asegurada a través de la evaluación estratégica de planes y programas que introdujo la Directiva 2001/42/CE, de 27 de junio, cuya trasposición se demoró en nuestro derecho hasta la promulgación de la Ley 9/2006, de 28 de abril, aunque ese retraso fue paliado por la jurisprudencia del Tribunal Supremo al considerar que la norma era suficientemente precisa e incondicionada para permitir su aplicación directa ${ }^{5}$.

La Ley estatal de Suelo integró desde el principio esta técnica en el procedimiento de elaboración de los instrumentos de ordenación territorial y urbanística (artículo 15.1 del texto inicial, hoy artículo 22.1 del Texto Refundido de 2015), lo que «facilita la incorporación de los criterios de sostenibilidad en la toma de decisiones estratégicas», como comienza diciendo el preámbulo de la nueva Ley 21/2013, de 9 de diciembre, de Evaluación ambiental, que ha sustituido a la anterior de 2006 y ha incorporado también a nuestro derecho la Directiva 2011/92/UE, de 13 de diciembre, de evaluación de las repercusiones de determinados proyectos públicos y privados sobre el medio ambiente y urbanística.

La Ley de Evaluación ambiental de 9 de diciembre de 2013 es, por su parte, determinante al respecto cuando en su artículo 9.1, párrafo segundo, establece que «carecerán de validez los actos de adopción, aprobación o autorización de los planes, programas y proyectos que... no se hayan sometido a evaluación ambiental», cuya falta de emisión en los plazos legalmente establecidos «en ningún caso podrá entenderse que equivale a una evaluación ambiental favorable», según precisa el artículo 10.

5 Vid. en este sentido la Sentencia de 8 de octubre de 2013. 
Hoy ya es sencillamente indiscutible, por lo tanto, que a la hora de ordenar un territorio determinado hay que tomar en consideración las distintas alternativas disponibles, los potenciales impactos ambientales de cada una de ellas y la posibilidad de minimizarlos o corregirlos, todo lo cual debe ser evaluado con sumo cuidado en función de los criterios de sostenibilidad ambiental que las Leyes establecen con el fin de justificar cómo y por qué se ha elegido finalmente una de esas alternativas. La jurisprudencia más reciente del Tribunal Supremo ya no se conforma fácilmente en este punto con simples palabras ${ }^{6}$.

Todo esto está ya asimilado y no llama la atención a estas alturas. Pero hay más y merece la pena reparar en ello. Me refiero a la sostenibilidad económica de la ordenación, extremo éste al que no se ha prestado hasta ahora la atención que merece y en el que por ello es obligado detenerse ahora.

\section{LA EXIGENCIA ADICIONAL DE SOSTENIBILIDAD ECONÓMICA}

El apartado 4 del artículo 22 del vigente Texto Refundido de la Ley de Suelo y Rehabilitación Urbana (artículo 15.4 del texto inicial de 2007) establece, en efecto, que «la documentación de los instrumentos de ordenación de las actuaciones de transformación urbanística deberá incluir un informe o memoria de sostenibilidad económica, en el que se ponderará, en particular, el impacto de la actuación en las Haciendas Públicas afectadas por la implantación y el mantenimiento de las infraestructuras necesarias o la puesta en marcha y la prestación de los servicios resultantes, así como la suficiencia y adecuación del suelo destinado a usos productivos».

Es ésta, sin duda, una exigencia fundamental, que, si efectivamente se respeta, puede cambiar los hábitos viciados de nuestro urbanismo. Porque hasta ahora todo era muy sencillo: el Legislador en cada intervención incrementaba las cesiones gratuitas a realizar por los propietarios, lo que aseguraba a los ayuntamientos la disponibilidad del suelo necesario para todo tipo de infraestructuras y dotaciones, cuya ejecución material corría también a cargo de aquéllos por costosa que fuera. De ese modo legisladores y gestores podían presumir de «progresistas» y de luchadores implacables contra la especulación, aunque la realidad era muy distinta, ya que el coste de esas cesiones en apariencia gratuitas y de la propia urbanización de los terrenos no era soportado realmente

\footnotetext{
${ }^{6}$ Vid. la Sentencia de 19 de diciembre de 2013.
} 
por propietarios y promotores, que, como es obvio, repercutían de inmediato en el precio de los solares todos los costes soportados en el curso del proceso de urbanización, lo que terminaba trasladándose al precio de las viviendas, cuyo comprador es el que finalmente pagaba, hipoteca mediante, la factura.

El idílico panorama inicial se oscurecía bruscamente, por lo demás, cuando, una vez ejecutada la urbanización, el Ayuntamiento correspondiente tenía que afrontar la tarea de mantener ésta en condiciones. Las zonas verdes públicas con tanto empeño exigidas al promotor resultaban entonces demasiado grandes porque generaban un gasto inasumible en concepto de jardinería y limpieza de las mismas; las extensas urbanizaciones de baja densidad obligaban a prolongar a pérdida las líneas municipales de autobuses cuando no las del propio ferrocarril metropolitano, lo que son palabras mayores, las «modernas» urbanizaciones subterráneas se transformaban en recogederos de basura y nidos de delincuencia, etc, etc. Hablo de cosas que veo/vemos todos los días y que tienen su origen en un planeamiento irresponsable, que sólo ha pensado en el diseño inicial de la ordenación, pero no en la sostenibilidad de la urbanización resultante de ese diseño.

La reciente Sentencia del Tribunal Supremo de 27 de octubre de 2015 explica muy bien en qué consiste el informe o memoria de sostenibilidad económica del planeamiento al que se refiere el artículo 22.4 del Texto Refundido de 2015 y en qué se diferencia de la memoria relativa a la viabilidad económica de la ordenación de la que se ocupa el apartado siguiente del mismo artículo, por lo que me ahorraré mayores explicaciones transcribiendo uno de sus pasajes. Dice así:

Conviene aclarar que el concepto de sostenibilidad económica a que se refiere el legislador estatal en el artículo 15.4 del Texto Refundido de la Ley de Suelo no debe confundirse con el de viabilidad económica, más ligado al sentido y finalidad del estudio económico-financiero, sino que va relacionado con dos aspectos distintos, la justificación de la suficiencia del suelo productivo previsto y el análisis del impacto de las actuaciones previstas en las Haciendas de las Administraciones Públicas intervinientes y receptoras de las nuevas infraestructuras y responsables de los servicios resultantes. Por otra parte, desde una perspectiva temporal el informe de sostenibilidad económica ha de considerar el coste público del mantenimiento y conservación de los nuevos ámbitos resultantes en función de los ingresos que la puesta en marcha de la actuación vaya a generar para las arcas de la Administración de que se trate. Es decir, mientras el estudio económico-financiero preverá el coste de ejecución de la actuación y las fuentes de financiación de la 
misma, el análisis de sostenibilidad económica no se ha de limitar a un momento o período temporal limitado, sino que ha de justificar la sostenibilidad de la actuación para las arcas públicas desde el momento de su puesta en marcha y en tanto siga generando responsabilidad para la Administración competente respecto de las nuevas infraestructuras y servicios necesarios. En definitiva, el estudio económico debe demostrar la viabilidad económica de una intervención de ordenación detallada en un sector o ámbito concreto y el informe o memoria de sostenibilidad económica debe garantizar analíticamente que los gastos de gestión y mantenimiento de las infraestructuras y servicios en ese sector o ámbito espacial pueden ser sustentados por las Administraciones públicas, en especial la Administración local competente en la actividad urbanística.

La Ley y la jurisprudencia imponen, pues, la sensatez a la hora de elaborar el planeamiento y exigen acreditarla con carácter previo a la aprobación de éste. Es, sin duda, un buen ejemplo en todos los sentidos porque la exigencia de un informe o memoria de sostenibilidad económica es anterior a la crisis y a la respuesta que a ésta quiso dar la reforma del artículo 135 de la Constitución en septiembre de 2011, la promulgación de la Ley Orgánica 2/2012, de 27 de abril, de Estabilidad Presupuestaria y Sostenibilidad Financiera, que vino a desarrollarlo y la firma y posterior ratificación del Tratado de Estabilidad, Coordinación y Gobernanza en la Unión Económica y Monetaria publicado en el Boletín Oficial del Estado de 2 de febrero de 2013 que forman ahora el marco general dentro del cual ha de desenvolverse la actividad de todas las Administraciones públicas y de las entidades y organismos de ellas dependientes.

\section{LA NECESARIA EXTENSIÓN AL CONJUNTO DE LOS SERVICIOS Y ACTIVIDADES DE LAS ADMINISTRACIONES PÚBLICAS DEL PRINCIPIO GENERAL DE SOSTENI- BILIDAD FINANCIERA}

A la disciplina presupuestaria y financiera establecida por las tres grandes normas que acabo de recordar he hecho ya alguna referencia en un escrito anterior ${ }^{7}$. No voy, pues, a insistir en ello en este momento.

Sí quiero, en cambio, hacer hincapié en los principios generales que la Ley Orgánica 2/2012, de 27 de abril, consagra en sus primeros artícu-

7 T. R. FERNÁNDEZ (2015), «La asistencia financiera a España desde la perspectiva del Derecho Administrativo», Themis, Revista da Faculdade de Direito da UNL, 5, págs. 17-38, que recoge los trabajos presentados al X Coloquio Luso-Español de Profesores de Derecho Administrativo en marzo de 2015. 
los y, concretamente, en los de sostenibilidad financiera y eficacia en la asignación y utilización de los recursos públicos, que conceptualmente están estrechamente emparentados.

Al primero de ellos se refiere el artículo 4 de la Ley, según el cual «las actuaciones de las Administraciones públicas y demás sujetos comprendidos en el ámbito de aplicación de esta Ley estarán sujetos al principio de sostenibilidad financiera», entendiendo por tal «la capacidad para financiar compromisos de gasto presentes y futuros dentro de los límites de déficit y deuda pública conforme a lo establecido en esta Ley en la normativa europea».

Todas las Administraciones públicas, esto es la Administración central, las comunidades autónomas, las corporaciones locales y las Administraciones de la Seguridad Social, así como «el resto de las entidades empresariales, sociedades mercantiles y demás entes de derecho público dependientes de las Administraciones públicas», están sujetas a este principio. Sobre esto no hay discusión alguna dados los términos del artículo 2 de la Ley.

¿En qué medida lo están? Aquí, en cambio, la opinión de la doctrina no es unánime ${ }^{8}$. Algún autor ha entendido, en efecto, que el principio es referible únicamente a la sostenibilidad de la Hacienda pública en su conjunto y no a cada servicio o actividad en particular, «lo cual permite que algún servicio sea insostenible». Yo no niego, desde luego, esto último porque el principio de sostenibilidad que la Ley formula es sólo eso, un principio, que puede entrar en conflicto con otro en un caso determinado y terminar generando una regla particular para ese caso concreto, pero dejando a un lado este asunto, que pertenece a la Teoría General del Derecho, creo que el principio en cuestión puede y debe ser referido a todos y cada uno de los servicios públicos, entendida esta expresión como alusiva a la actividad administrativa en general.

Me autoriza a pensarlo así el propio tenor literal del artículo 4.1 de la Ley, que se refiere a «las actuaciones de las Administraciones públicas y demás sujetos comprendidos en el ámbito de aplicación» de la misma sin excluir ninguna de ellas.

En esos mismos términos se pronuncia el artículo 7 de la Ley cuando alude al principio de eficiencia en la asignación y utilización de los recursos públicos. El apartado 2 del mismo se refiere, en efecto, a la gestión de los recursos públicos en general y exige a estos efectos la aplicación de «políticas de racionalización del gasto y de mejora de la ges-

8 Vid. recientemente el artículo de F. J. Villar RoJas (2016), «Principios de sostenibilidad y estabilidad presupuestaria en la gestión de los servicios públicos locales», El Cronista del Estado Social y Democrático de Derecho, 58-59, págs. 96 y ss. 
tión del sector público», a cuyo efecto el apartado 3 del propio precepto establece que «las disposiciones legales y reglamentarias, en su fase de elaboración y aprobación, los actos administrativos, los contratos y los convenios de colaboración, así como cualquier otra actuación... que afecte a los gastos o ingresos públicos presentes o futuros, deberán valorar sus repercusiones y efectos y supeditarse de forma estricta al cumplimiento de las exigencias de los principios de estabilidad presupuestaria y sostenibilidad financiera».

El texto transcrito es, pues, absolutamente concluyente. Toda disposición administrativa, todo acto, contrato o convenio, toda actuación de cualquier clase que sea exige una previa valoración de sus repercusiones y efectos en el plano presupuestario y financiero.

El artículo 129 de la novísima Ley de Procedimiento Administrativo Común de 1 de octubre de 2015 ratifica este planteamiento al referirse a los principios de buena regulación que deben observarse en el ejercicio de la iniciativa legislativa y la potestad reglamentaria, observancia que habrá de quedar «suficientemente justificada» en las exposiciones de motivos y preámbulos de los anteproyectos y proyectos correspondientes.

El apartado 6 del precepto dispone en concreto que «en aplicación del principio de eficiencia, la iniciativa normativa debe evitar cargas administrativas innecesarias o accesorias y racionalizar en su aplicación la gestión de los recursos públicos» y el apartado 7 añade por su parte que "cuando la iniciativa normativa afecte a los gastos e ingresos públicos, presentes o futuros, se deberán cuantificar y valorar sus repercusiones y efectos y supeditarse al cumplimiento de los principios de estabilidad presupuestaria y estabilidad financiera».

No podría ser de otro modo, ya que sería rigurosamente incoherente que el ordenamiento jurídico exigiera para los planes de ordenación urbana la elaboración de un informe o memoria de sostenibilidad económica con objeto de ponderar «el impacto de la actuación en las Haciendas Públicas afectadas por la implantación y el mantenimiento de las infraestructuras necesarias y la prestación de los servicios resultantes» (artículo 22.4 del Texto Refundido de la Ley de Suelo y Rehabilitación Urbana de 30 de octubre de 2015) so pena de nulidad de los planes en cuestión y, sin embargo, no reclamara otro tanto cuando se trata de decidir acerca de la construcción de una obra pública, de la puesta en marcha de un nuevo servicio público o, en fin, de la aprobación de una disposición de carácter general que establece unos determinados procedimientos y comporta para la o las Administraciones públicas concernidas mayores exigencias. 
Entiendo por ello que la Ley 27/2013, de 27 de diciembre, de Racionalización y Sostenibilidad de la Administración Local ha acertado plenamente al establecer en la nueva redacción que ha dado al artículo 86 de la Ley de Bases del Régimen Local que en el expediente acreditativo de la conveniencia y oportunidad del concreto ejercicio de la iniciativa pública para el desarrollo de actividades económicas se justifique que dicha iniciativa no genera riesgo para la sostenibilidad financiera del conjunto de la Hacienda municipal, a cuyos efectos - dice el preceptohabrá de «contener un análisis del mercado, relativo a la oferta y a la demanda existente, a la rentabilidad y a los posibles efectos de la actividad local sobre la concurrencia empresarial».

Y me parece bien igualmente que el nuevo apartado 2 del artículo 85 exija ahora que los servicios públicos locales se gestionen «de la forma más sostenible y eficiente» de las que el propio precepto enuncia y que a estos efectos dé preferencia a la gestión directa o al organismo autónomo local sobre la entidad pública empresarial local o la sociedad mercantil local, salvo que se justifique concretamente lo contrario.

Y, en fin, tampoco me parece mal que al referirse a la gestión indirecta de los servicios públicos locales «mediante las distintas formas previstas para el contrato de gestión de servicios públicos en el texto refundido de la Ley de Contratos del Sector Público» el precepto citado no exija memoria justificativa alguna de la decisión de elegir la vía contractual, ya que la adjudicación del contrato correspondiente habrá de serlo a través de la correspondiente licitación pública y esto asegura, por hipótesis, la obtención de la solución económicamente más ventajosa ${ }^{9}$.

Ese es, me parece, el camino a seguir ante cualquier tipo de actuación nueva o de reconsideración de las que ya estén en curso: valorar no sólo si se está en condiciones de afrontar los gastos de primer establecimiento y puesta en marcha, sino también y sobre todo si se cuenta con capacidad bastante para sostener su funcionamiento a un nivel razonable en el inmediato futuro. Con eso nos ahorraríamos por lo menos el patético espectáculo, tan frecuente por desgracia, de obras faraónicas que se han quedado en un cascarón vacío o poco menos porque no hay recursos bastantes para llenarlas de vida. Así lo he dicho ya en una ocasión anterior y no me importa repetirlo ahora con la esperanza de que el principio de sostenibilidad financiera que ha empezado a despuntar encuentre en la doctrina y en la jurisprudencia el apoyo que merece para lograr consolidarse y poder contribuir a la construcción de una Administración más racional y más eficaz.

\footnotetext{
9 Vid. en contra ibid.
} 